\title{
On Modeling the Quality of Nutrition for Healthy Ageing using Fuzzy Cognitive Maps
}

\author{
Sofia B. Dias ${ }^{1}$, Sofia J. Hadjileontiadou ${ }^{2}$, José A. Diniz ${ }^{1}$, João Barroso ${ }^{3}$, and \\ Leontios J. Hadjileontiadis ${ }^{4}$ \\ ${ }^{1}$ Faculdade de Motricidade Humana, Universidade de Lisboa, 1499-002 Cruz Quebrada, \\ Lisbon, Portugal \\ \{sbalula, jadiniz\}@fmh.ulisboa.pt \\ ${ }^{2}$ Hellenic Open University, Praxitelous 23, GR-10562, Athens, Greece \\ shadjileontiadou@gmail. com \\ ${ }^{3}$ INESC TEC and Universidade de Trás-os-Montes e Alto Douro, Vila Real, Portugal \\ jbarroso@utad.pt \\ ${ }^{4}$ Department of Electrical and Computer Engineering, Aristotle University of Thessaloniki GR- \\ 54124 Thessaloniki, Greece \\ leontiosdauth.gr
}

\begin{abstract}
Modelling dietary intake of older adults can prevent nutritional deficiencies and diet-related diseases, improving their quality of life. Towards such direction, a Fuzzy Cognitive Map (FCM)-based modelling approach that models the interdependencies between the factors that affect the Quality of Nutrition $(\mathrm{QoN})$ is presented here. The proposed FCM-QoN model uses a FCM with seven input-one output concepts, i.e., five food groups of the UK Eatwell Plate, Water (H2O), and older adult's Emotional State (EmoS), outputting the QoN. The weights incorporated in the FCM structure were drawn from an experts' panel, via a Fuzzy Logic-based knowledge representation process. Using various levels of analysis (causalities, static/feedback cycles), the role of EmoS and $\mathrm{H} 2 \mathrm{O}$ in the QoN was identified, along with the one of Fruits/Vegetables and Protein affecting the sustainability of effective food combinations. In general, the FCM-QoN approach has the potential to explore different dietary scenarios, helping health professionals to promote healthy ageing and providing prognostic simulations for diseases effect (such as Parkinson's) on dietary habits, as used in the H2020 iPrognosis project (www.i-prognosis.eu).
\end{abstract}

Keywords: Older Adults, Healthy Ageing, Emotional State, Fuzzy Cognitive Maps (FCMs), Quality of Nutrition (QoN), H2020 i-Prognosis.

\section{Introduction}

The ageing process is intrinsically complex, driven by multiple causal mechanisms. Ageing affects people in different ways, with a wide variation in age-related physical and mental functioning; healthier ageing, however, can be reached through modifying some lifestyle factors, e.g., being more physically active and/or eating a balanced diet. 
More specifically, ageing is determined by complex interactions between biological, environmental, sociocultural and economic factors. Nevertheless, factors that contribute to the ageing process, for instance poor nutrition and psychosocial characteristics (e.g., stress), can be modifiable [1]. This includes health and social care professionals, collaborating to (re)educate patients on improving their nutritional intake, as well as monitoring their ongoing nutritional status [2]. Despite significant medical advances, malnutrition remains a significant public health problem of developed countries. There is evidence that the nutritional intake of older people living in care homes is suboptimal, with high levels of saturated fat, salt, and added sugars [3]. Moreover, malnutrition has significant negative impacts on the physical and emotional well-being of older people, including depression, anxiety, and decreased quality of life (QoL) [4]. Optimizing the dietary intake of older adults, though, can prevent nutritional deficiencies and diet-related diseases, improving their QoL. Used by researchers for designing "ideal" diets, the UK Eatwell Plate is basically a pie-chart diagram consisting of five food group segments, the proportions of which are based on the dietary reference values for the population, namely: (i) bread, rice, potatoes, pasta and other starchy foods (Starchy) (33\% of the diet); (ii) fruit and vegetables (F\&V) (33\%); (iii) milk and dairy foods (Dairy) (15\%); (iv) meat, fish, eggs, beans and other non-dairy sources of protein (Protein) $(12 \%)$ and (v) foods and drinks that are high in fat or sugar, or both (HFHS) (8\%) [5-7].

In an effort to create a model that could incorporate the interdependencies between the five food groups of the UK Eatwell Plate, taking also into account two additional factors, i.e., water $(\mathrm{H} 2 \mathrm{O})$ and the emotional state $(\mathrm{EmoS})$ of the older adults, a FuzzyLogic (FL)-based approach is proposed here as a means to provide a generalized measure of the Quality of Nutrition (QoN). In particular, from the methodological point of view, the Fuzzy Cognitive Map (FCM) modelling methodology, introduced by Kosko [8], is adopted here, resulting in the FCM-QoN model. The latter is based on a directed weighted graph consisting of nodes and weighted arcs, acting as a general model for causes and effects, which is derived from cognitive maps, resulting of integration/combination of FL and neural network, which takes the experts' opinions as input and generates the maps [9]. The expert knowledge for the construction of the proposed FCMQoN model was drawn from a professional panel, involving Portuguese nutrition/diet experts. Various levels of analysis within the FCM-QoN scheme have shown that the proposed model can provide concepts interconnection and causal dependencies representation, contributing to the analysis and behavioural modeling of beneficial dietary habits and emotional states in the field of intelligent early detection and healthy ageing. The latter can be used as paradigms that comply with the scenarios of the i-Prognosis project (www.i-prognosis.eu), which is situated in the area of healthy and active ageing, targeting intelligent early detection and intervention in Parkinson's disease area.

The rest of the paper is constructed as follows: first, a review of the conceptual background related with healthy ageing and the main methodological issues of the FCM is presented, followed by data characteristics and a description of the proposed model. Next, a description of the implementation issues, analysis of the results, along with discussion and interpretation of the findings are provided. Finally, conclusions and future work conclude the paper. 


\section{Background and Methodology}

\subsection{Nutrients interdependencies with healthy ageing and the Eatwell Plate}

In general, the nutritional requirements of the older adult are the same as those for the rest of the adult population; however, some specific nutrients interdependencies should be considered, so to form the basis of some recommendations with related interventions that could assist healthy ageing. Focusing at specific nutrient characteristics the following could be noticed:

- FAT: Increased fat intakes are associated with higher levels of overweight and obesity, cardiovascular disease and some forms of cancer and diabetes mellitus. The older population are already at a higher risk of developing these conditions and high fat intakes can intensify this further. This is particularly so for saturated fat (animal type fats) with unsaturated fats (pure vegetable type fats) not being associated with the same risk. From this perspective, older people should eat less fat and in particular less saturated fat [10].

- PROTEIN: As muscle mass decreases in older people they should intake higher protein per kg lean body mass compared to younger adults; however, older people should therefore not eat excessive amounts of protein and their meals should be based around starchy foods [11].

- FIBER (Non-starch polysaccharide (NSP)): People who have a tendency to constipation are particularly encouraged to increase their NSP intake. As a result of slower gut movement, decreased activity levels and side effects of medications, some older people could be considered to be a group who may have a tendency to constipation, thus it is recommended that older people should eat more fiber [12].

- WATER: Older people are at increased risk of dehydration for a number of reasons including increased losses through skin as a result of skin becoming thinner, diminished ability of the kidneys to concentrate urine and a less sensitive thirst mechanism [13]. The effects of dehydration in older people which may impact on deterioration in nutritional status include: increased risk of pressure sores, unpleasant taste in the mouth, drowsiness, confusion, constipation, and increased risk of urinary tract infections. The consequences of dehydration are many and varied and most of these consequences impact on dietary intake and nutritional status. It is therefore essential that fluid intake (e.g., 6-8 glasses per day) is considered when managing the nutritional status of older people.

The above nutrients interdependencies with healthy ageing are considered in the construction of the Eatwell Plate [7, 14]. The latter highlights the different types of food that make up a functional diet, and shows the proportions needed to have a healthy, balanced diet (see Fig. 1). 


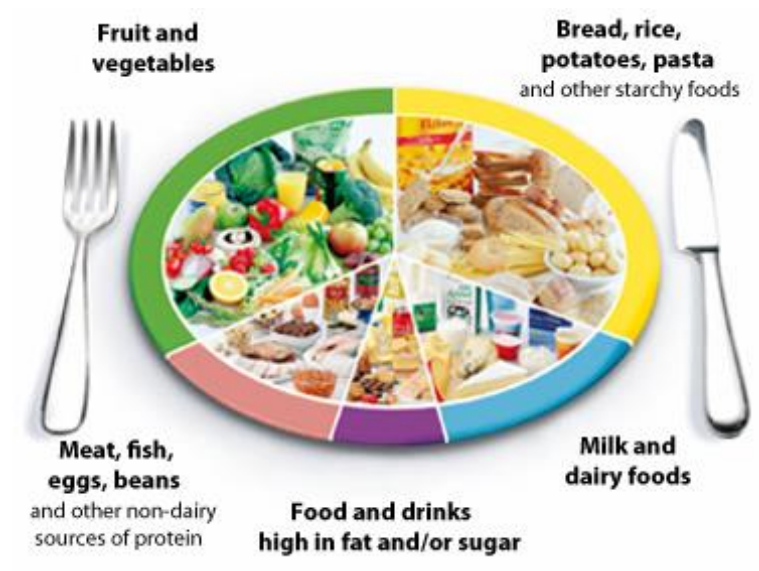

Fig. 1. The pie-chart diagram of UK Eatwell Plate consisting of five food group segments [5-7, 14].

The Eatwell Plate has been used as a reference with which the diets of different populations can be compared [15-17], for grouping foods in modelling the effects of increasing food prices on diet quality [18], and for designing "ideal" diets [19, 20]. To this end, the Eatwell Plate was used as a recommendation basis of the food group segments adopted here.

\subsection{The Fuzzy Cognitive Map (FCM) approach}

In a narrow sense, FL is considered a multi-valued logic technique that is imprecise or approximate. In other words, rather than using traditional logic theory where binary sets have a two-valued logic (i.e., true, 1 , and false, 0 ), fuzzy variables have a truthvalue between 0 (absolutely true) and 1 (absolutely false). In this way, using linguistic variables that describes the effect each factor in a knowledge system has on the others, FL can be used to convert the effects into values between 0 and 1. Actually, these values can be input into a graphical representation of the system containing all factors with directed lines (edges), revealing the calculated strength of the causal relationship between them. From this perspective, based on binary values, Kosko [8] suggested the use of fuzzy causal functions considering numbers within $[-1,1]$, modifying, in this way, the Axelrod's cognitive maps perspective and introducing the FCM concept.

In general, the FCM describes a cognitive map model with two main characteristics, namely: i) causal relationships between nodes are fuzzified; instead of only using signs to indicate positive/negative causality, a number is associated with the relationship to express the degree of relationship between two concepts; and ii) the system is dynamic involving feedback, where the effect of change in a concept node affects other nodes, which in turn can affect the node initiating the change.

FCMs have gained considerable research interest in several scientific fields from knowledge modelling to decision making [21, 22], resorting to data mining techniques 
for promoting user's expert knowledge [23, 24, 25] and modelling interdependence between concepts in the real-world, by graphically representing the causal reasoning relationships between vague or un-crisp concepts $[8,26,27]$. Overall, the FCM structure can be viewed as an artificial neural network, where concepts are represented by neurons and causal relationships by weighted links or edges connecting the neurons.

FCMs are graphical representations of the relationships between elements of a system, as perceived by "experts", where an expert is any person with knowledge of the system under scrutiny. FCM comprise vertices, representing concepts (C), joined by directional edges, representing causal relationships between concepts. Each connection is assigned a weight $w_{i j} \in[-1,1]$ which quantifies the strength of the causal relationship between concepts $C_{i}$ and $C_{j}$ [8]. A positive weight $w_{i j}>0$ indicates an excitatory relationship, i.e., as $C_{i}$ increases $C_{j}$ increases, while a negative weight $w_{i j}<0$ indicates an inhibitory relationship, i.e., as $C_{i}$ increases $C_{j}$ decreases.

\subsection{The proposed FCM-QoN model}

In this work, the FCM was initially constructed with the support of the knowledge and experience of the domain experts to determine the cause-effect relationships of the various knowledge representation elements (concept/nodes) of the system under study. In this way, the information regarding the factors/concepts that can contribute to healthy ageing field was gathered from nutritionists/dietitians and relevant literature based on recommendations and guidelines related to factors that contribute to the ageing process $[3-7,11,14,16]$. The gathered knowledge was presented to four nutritionists/dieticians affiliated to different centers/departments in Lisbon, Portugal. After some discussions/informal interviews with the experts, and taking into account the four aforementioned experts' opinion, eight concepts were identified as the main influencing factors that can contribute to the ageing process of older adults, in order to improve their QoL, namely: Starchy, F\&V, Dairy, Protein, HFHS, H2O, EmoS and QoN (output).

In this way, the involved FCM plays the role of a system representation, dismantling the 7 inputs-1 output relations, as represented by the estimated adjacency matrix. This actually reflects the expert's knowledge representation hidden in the IF/THEN fuzzy rules of the FCM-QoN model, yet in a more quantitative way, i.e., in the form of the interconnection weight values.

The eight concepts together form the basic nodes of the FCM-QoN model. The experts describe the influence of one concept on the other using fuzzy rules. In fact, different experts can have different opinions about the causal associations between different pair of concepts. From this perspective, in order to calculate the numerical weight that connects two nodes, the opinions of different experts are combined using an appropriate aggregation function, in order to eliminate subjectivity issues. The produced aggregated output is then defuzzified to rich a numerical weight of the link that connects two concepts; then, the weights of the links connecting the cause and effect nodes of the system are obtained, reflecting the combined opinion of the experts of causal associations between different attributes of the system [28]. 
In order to exemplify how the experts' opinions on causal relationships are converted into numerical weight values, the following fuzzy rule tries to express the interconnection between the concept HFHS and the output concept QoN:

"IF the value of the HFHS changes very much THEN the value of the QoN changes very much. Consequently, the effect of HFHS to QoN will be negatively very high."

Table 1. Construction of the triangle-membership functions and corresponding linguistic weights. Start, peak and end denote the triangle vertices.

\begin{tabular}{lccccccr}
\hline & \multicolumn{3}{c}{ Positively } & & \multicolumn{3}{c}{ Negatively } \\
\cline { 2 - 4 } \cline { 6 - 8 } Very low & Start & Peak & End & & Start & Peak & End \\
Low & 0.00 & 0.10 & 0.15 & & -0.15 & -0.10 & 0.00 \\
Medium & 0.10 & 0.20 & 0.30 & & -0.30 & -0.20 & -0.10 \\
High & 0.20 & 0.50 & 0.70 & & -0.70 & -0.50 & -0.20 \\
Very high & 0.60 & 0.70 & 0.80 & & -0.80 & -0.70 & -0.60 \\
Zero & 0.70 & 0.80 & 1.00 & & -1.00 & -0.80 & -0.70 \\
& 0.00 & 0.00 & 0.01 & & -0.01 & 0.00 & 0.00 \\
\hline
\end{tabular}

The MAX method for the aggregation of the triggered fuzzy membership functions were used. In general, six linguistic weights (i.e., very few, few, moderate, much, very much, zero) (see Table 1) and seven linguistic weights for the case of the Emotional State (i.e., positive2negative, positive2neutral, negative2positive, negative2neutral, neutral2positive, neutral2negative, no effect) were aggregated. Moreover, a seven-level of triangle membership functions $\mu$ (positively very low, positively low, positively medium, positively high, positively very high, zero, negatively very low, negatively low, negatively medium, negatively high, negatively very high) were adopted for the change effect within the range of $[-1,1]$ (see structural triangle characteristics in Table 1). Then the centroid defuzzification method were implemented to calculate the numerical value of the weight in the range $[-1,1]$ as illustrated in Fig. 2.

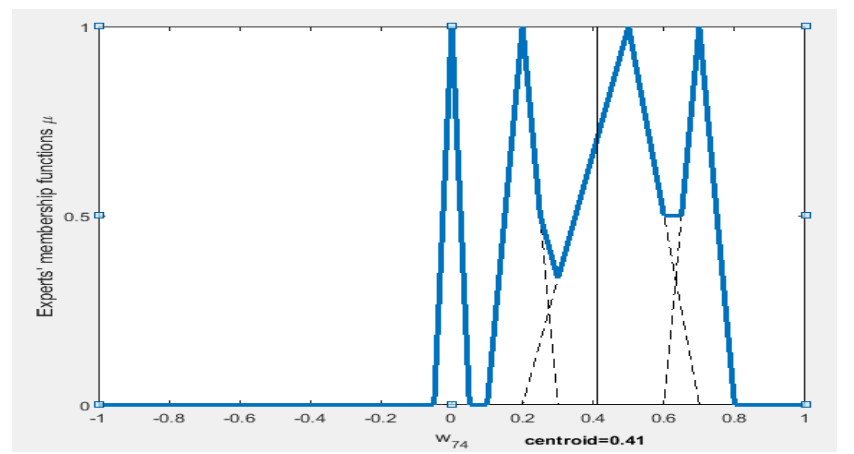

Fig. 2. The aggregation of linguistic variables using the MAX method, related with two concepts, namely: EmoS and Protein. The vertical line corresponds to the centroid of the pair (EmoS, Protein $)=0.41$, denoting weight $w_{74}$ (see also Table 2$)$. 


\subsection{Implementation Issues}

The implementation of the whole analysis of the FCM-QoN model was carried out in Matlab 2015a (The Mathworks, Inc., Natick, USA), using custom-made programming code. The visualization of the estimated FCM graph was carried out via a Matlab application, namely FCM-Viewer [29]. The results from the analysis of the aforementioned experts' opinions data with the proposed FCM-QoN model are described/discussed in the succeeding section.

\section{Results and Discussion}

\subsection{The derived FCM-QoN structure}

Figure 3 depicts the structure of the derived FCM-QoN model, showing the interconnection between the seven input concepts and the QoN output. The corresponding weights are analytically presented in Table 2 .

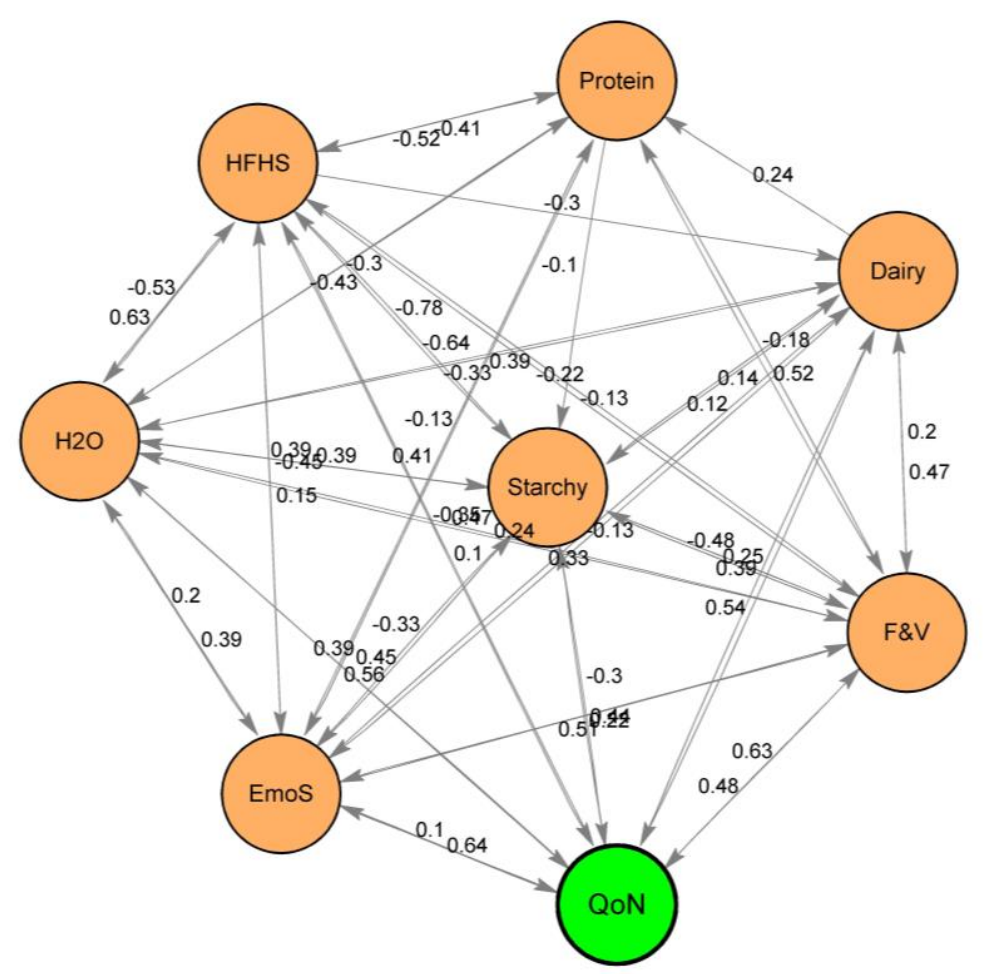

Fig. 3. The derived FCM-QoN model with the interconnection weights between the seven input concepts (in orange) and the output QoN (in green), shown in detail in Table 2. 
Table 2. The concept interconnection weights of the FCM-QoN model corresponding to the values of the FCM of the Fig. 3.

\begin{tabular}{lrrrrrrrr}
\hline & Starchy & F\&V & Dairy & Protein & HFHS & H2O & EmoS & QoN \\
\hline Starchy & 0 & -0.48 & 0.12 & 0 & -0.64 & 0.39 & -0.33 & -0.30 \\
F\&V & 0.25 & 0 & 0.47 & 0.52 & -0.13 & 0.24 & 0.22 & 0.63 \\
Dairy & 0.14 & 0.20 & 0 & 0.24 & 0 & 0.39 & -0.13 & 0.39 \\
Protein & -0.10 & -0.18 & 0 & 0 & -0.41 & -0.30 & -0.13 & 0 \\
HFHS & -0.78 & -0.22 & -0.30 & -0.52 & 0 & -0.53 & -0.45 & -0.35 \\
H20 & 0.39 & 0.47 & -0.33 & -0.43 & 0.63 & 0 & 0.20 & 0.39 \\
EmoS & 0.45 & 0.51 & 0.33 & 0.41 & 0.15 & 0.39 & 0 & 0.10 \\
QoN & 0.44 & 0.48 & 0.54 & 0 & 0.10 & 0.56 & 0.64 & 0 \\
\hline
\end{tabular}

\subsection{Analyzing scenarios}

For the constructed FCM-QoN model, a static analysis of the domain for establishing the relative importance of concepts, and indirect and total causal effects between concept nodes was conducted. In fact, several previous studies have used a static analysis for identifying important concepts based on network theory, such as centrality [30-32]. As a first level of analysis, the causal relationships between the concepts that are directly connected with the QoN, illustrated as orange circles in Fig. 3, are interpreted. In this line, the main concepts are Starchy, F\&V, Dairy, HFHS, H2O, EmoS, which are directly connected with the QoN (green circle). Based on the corresponding weights (Table 2), Protein is the only concept that does not (positively/negatively) directly affect the QoN, whereas the latter seems to affect the most (highest value (0.64) of the positive weights of Table 2) the EmoS, and then the $\mathrm{H} 2 \mathrm{O}$ ( 0.56 weight value). These findings indicate the need for attention in the way that emotional well-being of older people (e.g., depression, anxiety, optimism) should be considered as important factor in the field of healthy ageing combined with the adoption of healthy dietary habits. At the same time, due to the increased risk of dehydration in older people (older people lose around 2 liters of water per day), fluid intakes should be considered as part of intervention strategies; moreover, the increased risk of constipation in older people means that adequate fluid should be encouraged.

With regard to the highest negative effect, this appears between HFHS and F\&V $(-0.78)$, followed by the interdependency between Starchy and HFHS $(-0.64)$. These findings express the interconnection between the F\&V with HFHS and Starchy. In general, vegetables are good for healthy ageing, but some of them contain high levels of sugar. Knowing which vegetables have a high glycemic index number and therefore affect the blood sugar faster is valuable information. The higher the glycemic index, the more sugar the food contains and the faster blood sugar will feel the impact. Among the vegetables that are high in sugar, several fall into these categories, namely: roots, sweet peppers, bulbs and starchy vegetables.

As there is a number of interrelationships between the concepts within the FCM-QoN model, there is a potential for feedback cycles to exist within it, whereby a change in one concept can have a cumulative and/or self-sustaining effect. 
In this way, similarly to the work of [31], the second level of the static analysis was adopted, based on studying the characteristics of the weighted directed graph that represents the derived FCM-QoN, using graph theory techniques [33]. In this context, it is our intention to better understand the role of the influential factors of the QoN and to analyze their contribution to self-sustained cycles. From this perspective, the main feedback cycles (24) were identified within the estimated FCM-QoN model, structured by the concepts that are connected with the QoN. The identified main feedback cycles are tabulated in Table 3. Each identified feedback cycle was allocated a positive/negative sign (last column of Table 3), which was determined by multiplying the signs of the arcs present in each feedback cycle [33]. In fact, positive (negative) feedback cycle behavior is that of amplifying (counteracting) any initial change, leading to a constant increase (decrease) in case an increase is introduced to the system [33].

Table 3. The main feedback cycles (24) of the estimated FCM-QoN model (see Fig. 3). Note that these feedback cycles are structured by the concepts that are connected with the QoN.

\begin{tabular}{|c|c|c|}
\hline No. & Main Feedback Cycles & Cycle Sign \\
\hline 1 & Starchy $\rightarrow H 2 O \rightarrow E m o S \rightarrow Q o N \rightarrow F \& V \rightarrow$ Starchy & (+) positive \\
\hline 2 & Starchy $\rightarrow E m o S \rightarrow Q o N \rightarrow$ Starchy & (-) negative \\
\hline 3 & Starchy $\rightarrow$ Protein $\rightarrow F \& V \rightarrow$ QoN $\rightarrow$ Starchy & $(-)$ negative \\
\hline 4 & $F \& V \rightarrow E m o S \rightarrow Q o N \rightarrow F \& V$ & (+) positive \\
\hline 5 & $F \& V \rightarrow$ Dairy $\rightarrow$ Starchy $\rightarrow Q o N \rightarrow F \& V$ & (-) negative \\
\hline 6 & $F \& V \rightarrow H F H S \rightarrow H 2 O \rightarrow E m o S \rightarrow Q o N \rightarrow F \& V$ & $(+)$ positive \\
\hline 7 & Dairy $\rightarrow$ Protein $\rightarrow$ Starchy $\rightarrow$ QoN $\rightarrow$ Dairy & $(+)$ positive \\
\hline 8 & Dairy $\rightarrow H 2 O \rightarrow$ QoN $\rightarrow$ Dairy & $(+)$ positive \\
\hline 9 & Dairy $\rightarrow$ Protein $\rightarrow E m o S \rightarrow Q o N \rightarrow F \& V \rightarrow$ Dairy & $(-)$ negative \\
\hline 10 & Protein $\rightarrow$ HFHS $\rightarrow$ QoN $\rightarrow$ Dairy $\rightarrow$ Protein & $(+)$ positive \\
\hline 11 & Protein $\rightarrow F \& V \rightarrow$ QoN $\rightarrow$ Starchy $\rightarrow$ Protein & $(-)$ negative \\
\hline 12 & Protein $\rightarrow$ EmoS $\rightarrow$ QoN $\rightarrow H 2 O \rightarrow$ Protein & (+) positive \\
\hline 13 & $H F H S \rightarrow E m o S \rightarrow Q o N \rightarrow H F H S$ & $(-)$ negative \\
\hline 14 & $H F H S \rightarrow H 2 O \rightarrow E m o S \rightarrow Q o N \rightarrow$ Starchy $\rightarrow H F H S$ & $(+)$ positive \\
\hline 15 & $H F H S \rightarrow F \& V \rightarrow Q o N \rightarrow H F H S$ & (-) negative \\
\hline 16 & $\mathrm{H} 2 \mathrm{O} \rightarrow \mathrm{QoN} \rightarrow$ Dairy $\rightarrow \mathrm{H} 2 \mathrm{O}$ & $(+)$ positive \\
\hline 17 & $\mathrm{H} 2 \mathrm{O} \rightarrow \mathrm{EmoS} \rightarrow$ QoN $\rightarrow$ Starchy $\rightarrow \mathrm{H} 2 \mathrm{O}$ & (+) positive \\
\hline 18 & $\mathrm{H} 2 \mathrm{O} \rightarrow$ Protein $\rightarrow \mathrm{F} \& \mathrm{~V} \rightarrow \mathrm{QoN} \rightarrow \mathrm{H} 2 \mathrm{O}$ & (+) positive \\
\hline 18 & $E m o S \rightarrow Q o N \rightarrow F \& V \rightarrow E m o S$ & (+) positive \\
\hline 20 & EmoS $\rightarrow$ Starchy $\rightarrow$ Dairy $\rightarrow F \& V \rightarrow Q o N \rightarrow$ EmoS & $(+)$ positive \\
\hline 21 & $\mathrm{EmoS} \rightarrow \mathrm{H} 2 \mathrm{O} \rightarrow \mathrm{HFHS} \rightarrow$ Dairy $\rightarrow$ QoN $\rightarrow$ EmoS & $(-)$ negative \\
\hline 22 & $Q o N \rightarrow$ Dairy $\rightarrow F \& V \rightarrow H 2 O \rightarrow Q o N$ & (+) positive \\
\hline 23 & $Q o N \rightarrow E m o S \rightarrow$ Starchy $\rightarrow$ QoN & $(-)$ negative \\
\hline 24 & $Q o N \rightarrow H F H S \rightarrow F \& V \rightarrow Q o N$ & (-) negative \\
\hline
\end{tabular}

For example, the cycle No. $4(\mathrm{~F} \& \mathrm{~V} \rightarrow \mathrm{EmoS} \rightarrow \mathrm{QoN} \rightarrow \mathrm{F} \& \mathrm{~V})$ from Table 3 shows that F\&V affects EmoS, which affects QoN, which then affects back F\&V. This cycle could interpreted as follows: the consumption of F\&V could affect the EmoS, which could evoke the QoN, leading back to more consumption of F\&V. This cycle exhibits a positive sign (Table 3, last column) and shows how the feedback of FCM-QoN model could contribute in fueling a self-sustaining action $(\mathrm{F} \& \mathrm{~V})$, which has a cumulative effect on other actions, i.e., EmoS and QoN within the derived FCM-QoN model. 
In another example of Table 3, i.e., No. $21(\mathrm{EmoS} \rightarrow \mathrm{H} 2 \mathrm{O} \rightarrow \mathrm{HFHS} \rightarrow$ Dairy $\rightarrow$ QoN $\rightarrow$ $\mathrm{EmoS}$ ), it is clear that this feedback cycle is not self-sustained, as it is characterized by a negative sign (Table 3, last column), denoting that the combination of the food segments involved, and especially HFHS combined with Dairy, could lead to more negative emotions.

From an overall perspective of the cycles in Table 3, it seems that there is a balance between the number of cycles with positive (14) and negative (10) sign, denoting the opposite effect of various possibilities that could be adopted in the dietary habits. Consequently, following mainly self-sustained cycles, they potentially could promote further the QoN towards healthy nutrition in the silver community.

\section{Conclusion}

In this paper, a new way of modeling the food groups interconnection within the Eatwell Plate, combined with the water and emotional state, for the healthy ageing population was explored. Seen as a holistic and flexible model, the proposed FCM-QoN approach has the potential to surface possibilities and scenarios from different (macro, meso, micro) perspectives, helping nutritionists/dietitians and other health professionals to promote a healthy lifestyle, assisting older adults, in particular, to make better decisions regarding food choices as part of a healthy diet taking into account their emotional state. As a step further, the FCM-QoN model could be examined from a dynamic analysis approach, incorporating the effect of different diseases upon the dietary habits and emotional state, allowing for the simulations of the effects of the diseases on patients' nutrition behavior. This could also assist in the creation of prognostic indices related to the disease early symptoms. The latter is meticulously exploited in the H2020 i-Prognosis project (www.i-prognosis.eu), which is situated in the area of healthy and active ageing, targeting intelligent early detection and intervention in Parkinson's disease area.

Acknowledgements. This work has received funding from the EU H2020-PHC-20142015/H2020-PHC-2015, grant agreement N ${ }^{\circ}$ 690494: 'i-Prognosis' project (www.iprognosis.eu). Moreover, Dr. Dias (first author) acknowledges the financial support by the Foundation for Science and Technology (FCT, Portugal) (Postdoctoral Grant SFRH/BPD/496004/20) and the Interdisciplinary Centre for the Study of Human Performance (CIPER, Portugal). Finally, the authors would like to thank the four nutritionists/dieticians that served as experts in this study.

\section{References}

1. Myint, P.K., Welch, A.A.: Healthier ageing. BMJ, 344:e1214 (2012)

2. Taylor, C.: Looking at malnutrition from the patient's perspective. J Comm Nurs, 28(2):40$4(2014)$ 
3. Bamford, C., Heaven, B., May, C., Moynihan, P.: Implementing nutrition guidelines for older people in residential care homes: A qualitative study using normalization process theory. Implement Sci, 7:106 (2012)

4. Arvanitakis, M., Coppens, P., Doughan, L., Van Gossum, A.: Nutrition in care homes and home care: Recommendations - A summary based on the report approved by the Council of Europe. Clin Nutr, 28:492-496 (2009)

5. Food Standards Agency, Guidelines for use and reproduction of the Eatwell plate model, http://www. food.gov.uk/scotland/scotnut/eatwellplate/guidelines

6. Food Standards Agency, The Eatwell plate, http://tna.europarchive.org/ 20100929190231 /http: //www. eatwell.gov.uk/healthydiet/eatwellplate/

7. Public Health England in association with the Welsh government, the Scottish government and the Food Standards Agency in Northern Ireland, https : / /www . gov . uk/government/publications/the-eatwell-plate-how-to-use-it-in-promotional-material/

8. Kosko, B.: Fuzzy cognitive maps. Int J Man-Mach Stud, 24:65-75 (1986)

9. Dickerson, J.A., Kosko, B.: Virtual worlds as fuzzy cognitive maps. In: IEEE Virtual Reality Annual International Symposium, pp. 471-477. IEEE Press, New York (1993)

10. Taba Taba Vakili, S., Nezami, B.G., Shetty, A., Chetty, V.K., Srinivasan, S.: Association of high dietary saturated fat intake and uncontrolled diabetes with constipation: Evidence from the National Health and Nutrition Examination Survey. Neurogastroenterol Motil, 27(10):1389-1397 (2015)

11. Department of Health (DH) Committee on Medical Aspects of Food Policy: Dietary reference values for food energy and nutrients for the United Kingdom. Report on health and social subjects, n. 41, London, HMSO (1991)

12. Gandell, D., Straus, S.E., Bundookwala, M., Tsui, V., Alibhai, S.M.: Treatment of constipation in older people. CMAJ, 185(8):663-6 (2013)

13. Hodak, S.P., Verbalis, J.G.: Abnormalities of water homeostasis in aging. Endocrinol Metab Clin N Am, 34(4):1031-1046 (2005)

14. Jones, J., Duffy, M., Coull, Y., Wilkinson, H.: Older people living in the community-nutritional needs, barriers and interventions: A literature review, Project Report, Scottish Government Social Research, Edinburgh (2009)

15. Macdiarmid, J., Kyle, J., Horgan, G., Loe, J., Fyfe, M., Johnstone, A., McNeill, G.: Livewell: A balance of healthy and sustainable food choices. Godalming: WWF-UK (2011)

16. Harland, J.I., Buttriss, J., Gibson, S.: Achieving eatwell plate recommendations: Is this a route to improving both sustainability and healthy eating? Nutr Bull, 37:324-343 (2012)

17. Department for Environment, Food and Rural Affairs (DEFRA): Family food 2014 report (2015)

18. Jones, N.R.V., Conklin, A.I., Suhrcke, M., Monsivais, P.: The growing price gap between more and less healthy foods: Analysis of a novel longitudinal UK dataset. PLoS ONE, 9(10):e109343 (2014)

19. Banks, J., Williams, J., Cumberlidge, T., Cimonetti, T., Sharp, D.J., Shield, J.P.H.: Is healthy eating for obese children necessarily more costly for families? Br J Gen Pract, 62:e1-e5 (2012)

20. Leslie, W.S., Comrie, F., Lean, M.E., Hankey, C.R..: Designing the eatwell week: The application of eatwell plate advice to weekly food intake. Public Health Nutr, 16:795-802 (2012)

21. Lee, S., Yang, J., Han, J.: Development of a decision making system for selection of dental implant abutments based on the fuzzy cognitive map. Expert Syst Appl, 39(14):11564$11575(2012)$ 
22. Groumpos, P.P., Karagiannis, I.E.: Mathematical modelling of decision making support systems using fuzzy cognitive maps. In: Glykas, M. (ed.), Business process management, vol. 444, pp. 299-337. Springer, Berlin Heidelberg (2013)

23. Hong, T., Han, I.: Knowledge-based data mining of news information on the Internet using cognitive maps and neural networks. Expert Syst Appl, 23(1):1-8 (2002)

24. Kotsiantis, S.B.: Use of machine learning techniques for educational proposes: A decision support system for forecasting students' grades. Artif Intell Rev, 37(4):331-344 (2012)

25. Hadjileontiadou, S.J., Dias, S.B., Diniz, J.A., Hadjileontiadis, L.J.: Fuzzy logic-based modeling in collaborative and blended learning. In: Advances in educational technologies and instructional design (AETID). IGI Global, Hershey, PA (2015)

26. Dickerson, J., Kosko, B.: Virtual worlds as fuzzy cognitive maps. Presence, 3(2):173-189 (1994)

27. Giles, B.G., Findlay, C.S., Haas, G., LaFrance, B., Laughing, W., Pembleton, S.: Integrating conventional science and aboriginal perspectives on diabetes using fuzzy cognitive maps. Soc Sci Med, 64(3):562-576 (2007)

28. Papageorgiou, E.I.: A new methodology for decisions in medical informatics using fuzzy cognitive maps based on fuzzy rule-extraction techniques. Appl Soft Comput, 11(1):500$513(2011)$

29. Dias, S.B., Hadjileontiadou, S.J., Hadjileontiadis, L.J., Diniz, J.A.: Fuzzy cognitive mapping of LMS users' quality of interaction within blended-learning environment. Expert Syst Appl, 42(21):7399-7423 (2015)

30. Khan, M.S., Quaddus, M.: Group decision support using fuzzy cognitive maps for causal reasoning. Group Decis Negot, 13(5):463-480 (2004)

31. Hossain, S., Brooks, L.: Fuzzy cognitive map modelling educational software adoption. Comput Educ, 51(4):1569-1588 (2008)

32. Yaman, D., Polat, S.: A fuzzy cognitive map approach for effect-based operations: An illustrative case. Inf Sci, 179(4): 382-403 (2009)

33. Tsadiras, A.K., Kouskouvelis, I., Margaritis, K.G.: Making political decisions using fuzzy cognitive maps: The FYROM crisis. In: Proceedings of the 8th Panhellenic conference on informatics (EPY' 2003), vol. 2, pp. 501-510. Thessaloniki, Greece (2001) 